\title{
Effect of the degree of gastric filling on the measured thickness of advanced gastric cancer by computed tomography
}

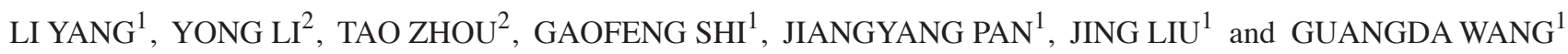 \\ ${ }^{1}$ Department of Computed Tomography and Magnetic Resonance; ${ }^{2}$ Department of Surgery, \\ The Fourth Hospital of Hebei Medical University, Shijiazhuang, Hebei 050011, P.R. China
}

Received March 15, 2017; Accepted January 25, 2018

DOI: $10.3892 / \mathrm{ol} .2018 .8907$

\begin{abstract}
Imaging of gastric cancer thickness is closely associated with the depth of tumor invasion, which provides guidance for clinical staging and assists the evaluation of the effects of adjuvant therapy. However, it is unclear whether the measurement of thickness is affected by the degree of gastric filling, and its accuracy and reliability are under-reported. The present study aimed to investigate the influence of the degree of gastric filling on the measurement of gastric cancer thickness. A total of 38 patients with advanced gastric cancer who underwent enhanced abdominal computed tomography (CT) scanning at the Department of CT and MR in The Fourth Hospital of Hebei Medical University (Shijiazhuang, China) between July and September 2016 were recruited, consisting of 21 newly diagnosed cases and 17 follow-up cases following non-surgical treatments. Plain scanning (prior to filling) and enhanced scanning in venous phase (following filling) were performed. Axial CT images prior to and following filling of the normal part of gastric wall and the lesions were compared. The same procedure was repeated on these participants 1 month later by the same radiologist, and the results were compared with those obtained previously. Normal gastric wall thickness prior to and following gastric filling was significantly different (all $\mathrm{P}<0.001)$ with the most substantial changes observed at the greater curvature. Lesion thickness prior to and following filling was similar in newly diagnosed patients, but significantly different in patients for re-examination $(\mathrm{P}<0.05)$. The two thickness measurements in the same patients were consistent. The measured thickness of gastric cancer in newly diagnosed patients was relatively stable, and could be used as an indicator in baseline CT examination. Maintaining a similar degree of gastric filling during re-examination could aid the accurate evaluation of treatment efficacy.
\end{abstract}

Correspondence to: Dr Yong Li, Department of Surgery, The Fourth Hospital of Hebei Medical University, 12 Jian-Kang Road, Shijiazhuang, Hebei 050011, P.R. China

E-mail: li_yong_hbth@126.com

Key words: X-ray computed tomography, stomach neoplasms, gastric wall, tumor thickness

\section{Introduction}

Gastric cancer, the fourth most common type of cancer worldwide, remains the second leading cause of cancer mortality. The incidence of gastric cancer is highest in Asia and certain parts of South America, particularly in China, Korea, and Japan (1,2). Over $90 \%$ of patients with gastric cancer in China were diagnosed in the advanced stage (3). Gastric cancer is characterized by high rates of incidence, metastasis, and mortality, and low rates of early diagnosis, radical resection, and 5-year survival (3).

Upper gastrointestinal imaging and gastroscopy are the most widely used methods for screening and diagnosing of gastric cancer; however, these methods have limited utility in evaluating the depth of invasion and the presence of extra-stomach metastases (1). Currently, the most commonly methods for imaging gastric cancer are computed tomography (CT) scanning and ultrasonic endoscopy (1). Ultrasonic endoscopy has been utilized widely to measure the depth of gastric wall invasion; however, this procedure appears to be less effective than $\mathrm{CT}$ when used to detect lymphatic and distant metastasis (4). Although CT is an indirect method for evaluating the presence gastric cancer and is not specific for early-stage gastric cancer, with advances in CT scanning and post-reconstruction technologies, CT has become an important method for the pre-operative staging and treatment efficacy evaluation in patients with gastric cancer who have already been diagnosed via electronic gastroscope (5-8). During CT examination, the thickness of the focal wall is one of the imaging measurements, as gastric thickening is one of the major manifestations of an advanced gastric cancer (1). The thickness of the focal gastric wall is closely associated with the depth of cancer invasion and Tumor-Node-Metastasis (TNM) stages (9), which assist the clinics to select an optimal treatment for early-stage or advanced gastric cancer. For example, if the tumor has invaded the serosa layer in a patient with gastric cancer, neoadjuvant chemotherapy may be recommended prior to surgery. Furthermore, the thickness of a gastric tumor could reflect the biological behaviors of tumor in a certain degree (10). Previous studies have indicated that the Response Evaluation Criteria in Solid Tumors are not applicable for gastrointestinal tumors, including gastric cancer, and that measuring the tumor volume and positron emission tomography/CT examination could reflect the treatment efficacy more accurately (11-13). 
However, according to our clinical experience, effective non-surgical treatments can lead to reduced gastric cancer thickness. Therefore, measuring the thickness of gastric cancer could have potential significance for clinical staging, clinical assessment and treatment efficacy evaluation. During CT imaging, gastric filling is often performed to ensure adequate distention, which is essential to ensure that areas of disease are not overlooked and to prevent the collapsed gastric wall from mimicking disease (14). Liquid, often water, is commonly used, but an alternative method is to introduce gas into the stomach $(2,15)$. The present study used anaerogenic powder to produce carbon dioxide gas for gastric filling (1). However, it remains unclear whether the measurement of gastric cancer thickness is affected by the degree of gastric filling. The accuracy and reliability of gastric cancer thickness measurements are rarely reported. Therefore, the clinical relevance of gastric cancer thickness remains uncertain; it is therefore necessary to understand whether the thickness of gastric cancer is affected by the degree of gastric filling, and to investigate the accuracy and reliability of the measurements.

The present study aimed to investigate the influence of gastric filling degree on the measurement of gastric cancer thickness.

\section{Patients and methods}

Subjects. Data from patients with gastric cancer that underwent enhanced abdominal CT scanning in the Department of MR of the Fourth Hospital of Hebei Medical University (Shijiazhuang, China) between July and September 2016 were prospectively included in the present study.

The inclusion criteria of the patients were as follows: i) Treatment-naïve and confirmed with gastric cancer by gastroscopic biopsy, but had not received any antitumor therapies, or were patients with gastric cancer that were undergoing re-examination following non-surgical antitumor treatments (such as radiotherapy, chemotherapy and oral targeting drugs); ii) without contraindications for the use of anisodamine hydrochloride, including glaucoma and urinary retention; iii) without contraindications for the use of iodine contrast medium; iv) without symptoms of gastrointestinal perforation; v) without contraindications for aerogenic powder; and vi) with advanced gastric cancer according to imaging staging, which had a thickness that was $50 \%$ higher than the thickness of adjacent normal gastric wall following filling.

The exclusion criteria were as follows: i) The patients had pyloric obstruction, abiding food was found in the abdominal cavity following food and water fasting, and the abdominal cavity was in dilation; and ii) the abdominal cavity was not well filled following oral intake of aerogenic powder, as evidenced by the duplicature continuing to gather together.

A total of 45 patients completed the examination. There were four patients with residual food in the abdominal cavity or in a state of dilation during plain scanning, and three patients in a sub-optimal filling state following the oral intake of aerogenic powder, who were excluded. Therefore, a final total of 38 patients ( 30 males, 8 females; mean age, $63.2 \pm 7.7$ years; age range, 37-76 years) were included in the present study.

The non-conventional clinical examination procedures used in the present study were approved by the Ethics Committee of the Fourth Hospital of Hebei Medical University. All the patients provided written informed consent.

CT scanning. A second-generation dual-source CT scanner (SOMATOM Definition Flash; Siemens Healthcare, Erlangen, Germany) was used to perform CT scanning. The patients were fasted from food and water for $6 \mathrm{~h}$ before the scan. During CT scanning, the patients were placed in a supine position and plain scanning was performed when the abdominal cavity was in the natural state of emptiness. The plain scanning covered the area between the diaphragmatic dome and pelvic floor. The parameters for the plain scanning were as follows: Scanning voltage, $120 \mathrm{Kv}$; current, $210 \mathrm{~mA}$; collimation width, $128 \times 0.6 \mathrm{~mm}$; and screw pitch, 0.9 . To reduce the tension of the gastric wall, the abdominal cavity was fully dilated, and to avoid gastrointestinal peristalsis artifacts, an intramuscular injection of anisodamine hydrochloride $(10 \mathrm{mg})$ was administered once the plain scanning was completed. Once dry mouth symptoms appeared, which indicated that the stomach muscle tension-lowering drugs were taking effect, $6 \mathrm{~g}$ aerogenic powder was administered orally. The patients were asked not to belch or talk, and then enhanced scanning was performed immediately. A high-pressure injector (CT-D; Medtron AG, Saarbrücken, Germany) was used to inject the contrast agent (iohexol; $300 \mathrm{mgI} / \mathrm{ml}$, dose of $2 \mathrm{ml} / \mathrm{kg}$ ) into the cubital fossa vein at a velocity of $3.0 \mathrm{ml} / \mathrm{sec}$. Next, dual-energy arterial phase and venous phase scanning were performed at 25 and $70 \mathrm{sec}$ after the injection of contrast agent, during which the patients were asked to hold the breath. The enhanced scanning covered the area between the diaphragmatic dome and lower gastric edge, and between the diaphragmatic dome and pelvic floor. The parameters for the enhanced scanning were as follows: Tubal voltages, $100 \mathrm{kV}$ and $\mathrm{Sn} 140 \mathrm{kV}$, with Care Dose 4D turned on; reference currents, 230 and $178 \mathrm{~mA}$; collimation width, $32 \times 0.6 \mathrm{~mm}$; and screw pitch, 0.55 .

The aforementioned protocol consisted of two steps. In the first step, the empty stomach in the natural state was scanned. In the second step, the stomach was filled at a low-tension state and then images were captured by enhancement scanning. The procedure was feasible and easy to operate without the increase of radiation dose. In the present study, the protocol was only utilized to investigate the association between the thickness of gastric lesions and the filling level.

Image analysis. The images obtained by plain scanning and in the venous phase were reconstructed. The plain scanning images were reconstructed to be $1.0 \mathrm{~mm}$ in thickness using the B10f algorithm (Siemens Healthineers, Erlangen, Germany); the fusion images at venous phase were reconstructed to be $1.0 \mathrm{~mm}$ in layer thickness with the B30f algorithm. The reconstructed images were input into a picture archive and retrieval system, and an investigator with 13 years of experience of imaging diagnosis was asked to measure the thickness and perform the staging classification.

The axial and multiplanar reconstruction images were used for imaging staging, according to the TNM staging system (7th edition) issued by the American Joint Committee on Cancer (AJCC) (16-18).

The different gastric areas were determined according to the 'three-area method' (19) prior to thickness measurement, 
which was in consistent with surgical stomach partition method. In brief, the cardia was present at the joint between stomach and esophagus; the antrum was the area between the angular incisure and the pylorus; and the gastric body was the area between cardia and antrum, which included the lesser and greater curvature sides. The thickness was measured as follows. i) The length-measuring tool (Syngo MMWP VE36A; Siemens Healthineers) was used for the measurement, with the assistance of adjusting window position and width, as well as local zoom in. The axial images in the venous phase were observed consecutively, and the layer with the highest thickness of gastric cancer following gastric filling was selected for measurement. For the patients with ulcerated cancer, the thickness of annular dikes was also measured. The areas adhering to the perigastric lymph nodes and the perigastric adipose tissues, with invasion involvement were not included in the measurement. ii) According to the position used in the measurement during the venous phase, the anatomical landmarks, including the cardia, pylorus, and ulcer floor, were used as the references to enable selection of the same position in the plain scanning images to measure the thickness prior to filling. The same method was used to measure the thickness of the gastric wall in the non-cancerous normal areas, of which the area was at least $3 \mathrm{~cm}$ to the margin of the gastric cancer. The thickness of the residual normal gastric wall at the cancerous area was not measured. For each patient, the thickness of the gastric cancer was measured again 1 month later, using the same imaging data. An interval of 1 month, was chosen for the following reasons: i) This length of time could minimize the influence of remembering details of the first measurement; and ii) re-examination of the gastric cancer patients who underwent a neoadjuvant therapy was generally following 2 cycles of chemotherapy and therefore, 1 month after the first examination.

The distance between the normal gastric wall and the cancer margin was at least $3 \mathrm{~cm}$, and the thickness of the gastric wall in the area of the cancer was not measured. For instance, the thickness of the normal wall of lesser gastric curvature was not measured for the patients with cancer of the lesser gastric curvature.

Statistical analysis. SPSS 21.0 (IBM Corp., Armonk, NY, USA) and MedCalc 16.2 (MedCalc Software bvba, Ostend, Belgium) were used for the statistical analysis. $\mathrm{P}<0.05$ was considered statistically significant. The quantitative data in normal distribution are depicted as the mean \pm standard deviation; a paired Student's t-test was used for the comparisons between two groups for quantitative date with equal variances. Quantitative data in non-normal distributions are depicted as medians and inter-quartile ranges, and compared with Wilcoxon signed ranks test. Qualitative data were described with frequencies and percentages, and compared with Fisher's exact test or non-parametric test following rank conversion. Consistency of the two measurements by the same investigator was evaluated by Bland and Altman plotting.

\section{Results}

General characteristics of the subjects. None of the patients experienced adverse effects, such as allergy and perforation, or complications. Certain patients reported abdominal fullness following the intake of the aerogenic powder, which was tolerable and disappeared following the spontaneous exhaust that occurred once the scanning was completed. Among these patients, 21 were newly diagnosed, and the other 17 were being re-examined following treatments (Table I). The clinical and demographic characteristics were similar between the two groups.

The stages of the 8 newly diagnosed patients that underwent surgical treatments were determined by postoperative pathological examination, whereas staging of the other patients was conducting using imaging. Among the 17 patients for re-examination, the treatments were as follows: Chemotherapy for 15 patients (including 4 using the S-1/oxaliplatin strategy, 3 using the oxaliplatin and capecitabine strategy, 3 used TO strategy, 1 using the 5-fluorouracil, folinic acid and oxaliplatin strategy, 1 using the ocetaxel and cisplatin strategy, 1 using oral intake of a tegafur-gimeracil-oteracil potassium capsule and 1 using an unknown chemotherapy regimen), radiotherapy for 1 patient, and interventional embolization for 1 patient. For the 21 newly diagnosed patients, 8 underwent surgical resection, 7 received chemotherapy, and the other 6 were not treated in The Fourth Hospital of Hebei Medical University. The consequent treatments for the last 6 patients were also unknown. The pathological types of the carcinoma in the 8 patients that underwent surgical resection were determined by postoperative pathological examination, whereas the pathological types of the others were pathological types of the biopsies.

Measurement thickness of the normal gastric walls prior to and following filling. The thickness of the normal gastric wall in 88 areas was obtained from the 38 patients with gastric cancer. The thickness of the normal gastric wall in each area prior to and following filling was significantly different $(\mathrm{P}<0.05)$. The change was greatest at the greater curvature (Table I; Fig. 1).

Measurement thickness of the gastric cancer prior to and following filling. A total of 32 measurements of the gastric cancer thicknesses were obtained from the 21 newly diagnosed patients. The difference in the thickness of the gastric cancer at different areas (except for those at the greater curvature, which were too few to provide enough data for statistical analysis) prior to and following filling was not statistically significant (P>0.05; Table II; Figs. 2 and 3).

For the 17 patients who were being re-examined following treatment, 31 measurements of the thickness of gastric cancer at different areas were obtained. The thickness of the gastric cancer at different areas (except for those at the greater curvature, which were too few to provide enough data for statistical analysis) prior to and following filling was statistically significant $(\mathrm{P}<0.05$; Table III; Figs. 4-6).

Consistency of measuring the gastric cancer thickness. The thicknesses of the gastric cancer at the 63 areas of the 38 patients were measured again 1 month later by the same investigator. According to Bland and Altman plots, two data points prior to the gastric filling $(2 / 63,3.17 \%)$ were outside the $95 \%$ confidential interval (CI) of limits of agreement (LoA). The mean value of the differences between the two measurements of the gastric cancer thicknesses was -0.06 
Table I. Clinical and demographic characteristics of the 38 patients.

\begin{tabular}{|c|c|c|c|c|}
\hline Characteristic & Total, n (\%) & $\begin{array}{c}\text { Newly } \\
\text { diagnosed, n (\%) }\end{array}$ & $\begin{array}{c}\text { Re-examination, } \\
\mathrm{n}(\%)\end{array}$ & $\begin{array}{l}\text { P-value (Newly diagnosed } \\
\text { vs. re-examination) }\end{array}$ \\
\hline \multicolumn{5}{|l|}{ Sex } \\
\hline Male & $30(78.95)$ & $15(71.43)$ & $15(88.24)$ & 0.257 \\
\hline Female & $8(21.05)$ & $6(28.57)$ & $2(11.76)$ & \\
\hline \multicolumn{5}{|l|}{ Age (years) } \\
\hline Mean \pm standard deviation & $63.2 \pm 7.7$ & $64.5 \pm 7.0$ & $61.7 \pm 8.4$ & 0.171 \\
\hline \multicolumn{5}{|l|}{ Anatomical location } \\
\hline Cardia/corpus/antrum & $2(5.26)$ & 0 & $2(11.76)$ & $0.661^{\mathrm{a}}$ \\
\hline Cardia/orpus & $13(34.21)$ & $8(38.10)$ & $5(29.41)$ & \\
\hline Corpus & $4(10.53)$ & $4(19.05)$ & 0 & \\
\hline Antrum & $6(15.79)$ & $4(19.05)$ & $2(11.76)$ & \\
\hline Cardia & $9(23.68)$ & $3(14.29)$ & $6(35.29)$ & \\
\hline Corpus/Antrum & $4(10.53)$ & $2(9.52)$ & $2(11.76)$ & \\
\hline \multicolumn{5}{|l|}{ Stage $^{\mathrm{a}}$} \\
\hline $\mathrm{T} 3$ & $5(13.16)$ & $3(14.29)$ & $2(11.76)$ & $1.000^{\mathrm{a}}$ \\
\hline $\mathrm{T} 4 \mathrm{a}$ & $28(73.68)$ & $15(71.43)$ & $13(76.47)$ & \\
\hline $\mathrm{T} 4 \mathrm{~b}$ & $5(13.16)$ & $3(14.29)$ & $2(11.76)$ & \\
\hline N0 & $8(21.05)$ & $4(19.05)$ & $4(23.53)$ & $0.701^{\mathrm{a}}$ \\
\hline N1 & $11(28.95)$ & $6(28.57)$ & $5(29.41)$ & \\
\hline $\mathrm{N} 2$ & $14(36.84)$ & $8(38.10)$ & $6(35.29)$ & \\
\hline N3 & $5(13.16)$ & $3(14.29)$ & $2(11.76)$ & \\
\hline M0 & $26(68.42)$ & $16(76.19)$ & $10(58.82)$ & 0.307 \\
\hline M1 & $12(31.58)$ & $5(23.81)$ & 7 (41.18) & \\
\hline \multicolumn{5}{|l|}{ Treatment } \\
\hline Surgical resection & $8(21.05)$ & $8(38.10)$ & 0 & $0.373^{\mathrm{a}}$ \\
\hline Chemotherapy & $22(57.89)$ & $7(33.335)$ & $15(88.24)$ & \\
\hline Radiotherapy & $1(2.63)$ & 0 & $1(5.88)$ & \\
\hline Others & $7(18.42)$ & $6(28.57)$ & $1(5.88)$ & \\
\hline \multicolumn{5}{|l|}{ Pathological type } \\
\hline Adenocarcinoma & $34(89.47)$ & $18(85.71)$ & $16(94.12)$ & $0.383^{\mathrm{a}}$ \\
\hline Well differentiated & 0 & 0 & 0 & \\
\hline Moderately differentiated & $5(14.71)$ & $5(27.78)$ & 0 & \\
\hline Poorly differentiated & $15(44.12)$ & $7(38.89)$ & $8(50.00)$ & \\
\hline Unknown & $14(41.18)$ & $6(33.33)$ & $8(50.00)$ & \\
\hline Signet ring cell carcinoma & $2(5.26)$ & $1(4.76)$ & $1(5.88)$ & \\
\hline Mucinous adenocarcinoma & $2(5.26)$ & $2(9.52)$ & 0 & \\
\hline
\end{tabular}

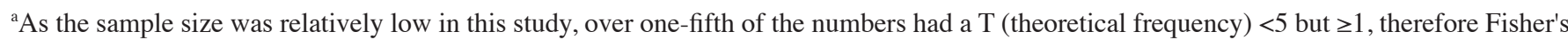
exact test could not be applied for the analysis. These data were analyzed by non-parametric test following rank conversion.

(95\% CI of the LoA, $-0.5943,0.4784)$. A total of three data points following gastric filling $(3 / 63,4.76 \%)$ were outside the $95 \%$ CI of LoA. The mean value of the differences between the two measurements of the gastric cancer thicknesses was -0.03 (95\% CI of the LoA, -0.6194, 0.5566). Over $95 \%$ of all the data points prior to and following filling were in the $95 \%$ CI of LoA, and the two measurements demonstrated high consistency (Figs. 7 and 8).

\section{Discussion}

The present study aimed to assess the influence of the degree of gastric filling on the measurement of gastric cancer thickness. The results revealed that the gastric wall thickness measurements prior to and following filling were significantly different, and the change was greatest at the greater curvature. The measurement of cancer thickness prior to and following 
Table II. Comparison of the measurement thickness of the normal gastric walls prior to and following filling.

\begin{tabular}{|c|c|c|c|c|c|}
\hline Area & Patients, $\mathrm{n}$ & Thickness prior to filling, $\mathrm{cm}^{\mathrm{a}}$ & Thickness post-filling, $\mathrm{cm}^{\mathrm{a}}$ & $\mathrm{Z}$ - or t-value & P-value \\
\hline Cardia & 14 & $0.89 \pm 0.23$ & $0.47 \pm 0.03$ & $9.751(\mathrm{t})$ & $<0.001$ \\
\hline Lesser curvature & 17 & $0.84(0.60,1.01)$ & $0.31(0.23,0.54)$ & $-3.622(\mathrm{Z})$ & $<0.001$ \\
\hline Greater curvature & 32 & $1.33(1.08,1.52)$ & $0.29(0.22,0.37)$ & $-4.937(\mathrm{Z})$ & $<0.001$ \\
\hline Antrum & 25 & $0.70(0.46,0.81)$ & $0.25(0.20,0.39)$ & $-4.286(\mathrm{Z})$ & $<0.001$ \\
\hline Total & 88 & $0.88(0.65,1.23)$ & $0.30(0.23,0.42)$ & $-8.101(\mathrm{Z})$ & $<0.001$ \\
\hline
\end{tabular}

${ }^{a}$ Data are presented as the mean \pm standard deviation or median $(95 \%$ confidence interval).

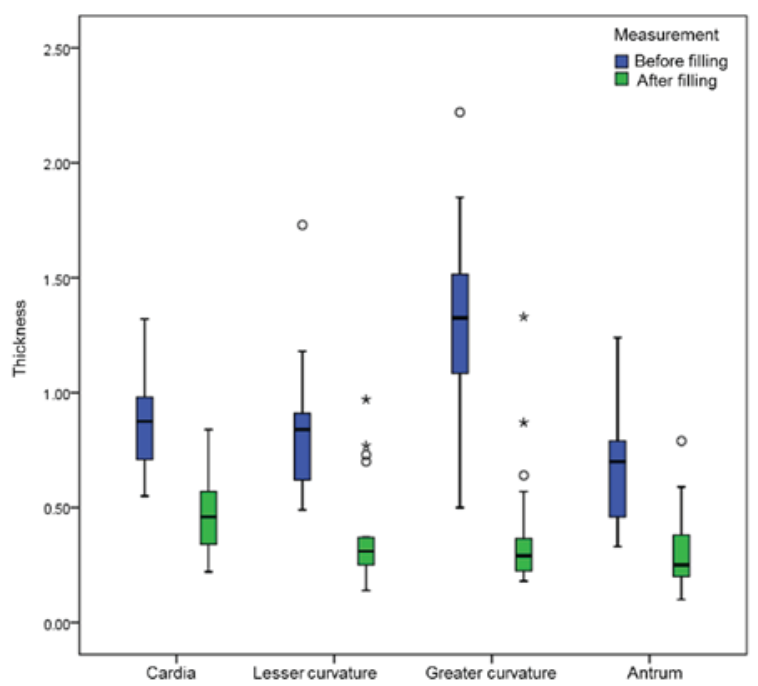

Figure 1. Thickness of the normal gastric wall at each area prior to and following gastric filling.

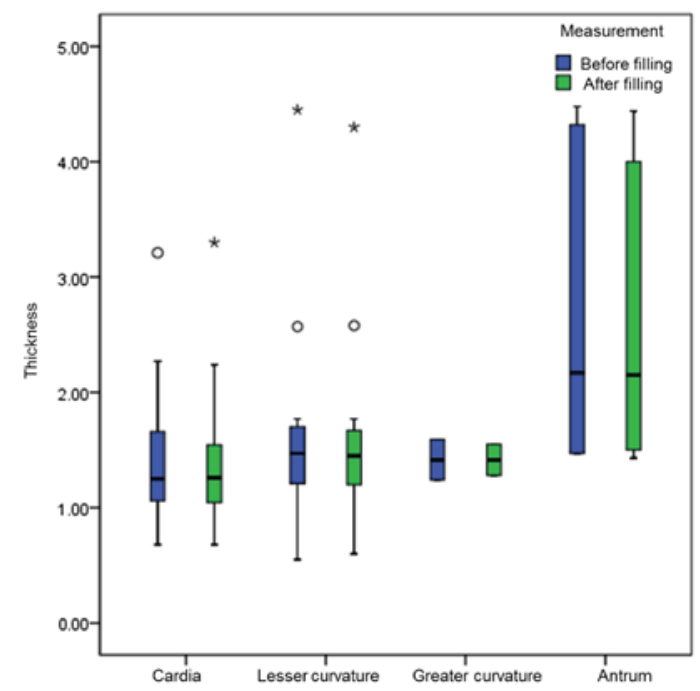

Figure 2. Thickness of the gastric cancer at each area prior to and following gastric filling in the newly diagnosed patients.

gastric filling was similar in newly diagnosed patients, but significantly different in patients undergoing re-examination. However, two thickness measurements, a month apart, in the same patients were consistent. To the best of our knowledge,
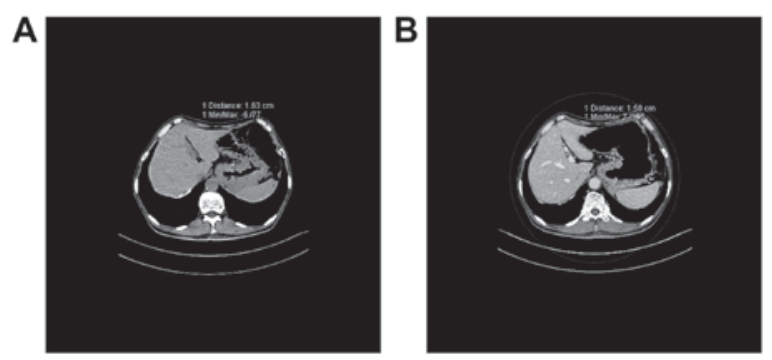

Figure 3. A 74-year-old male patient who was newly diagnosed with cardiac cancer. The thickness of the normal gastric wall at the greater curvature (A) prior to and (B) following filling was 1.39 and $0.29 \mathrm{~cm}$, respectively, which were significantly different. The thickness of the cardiac cancer was 1.63 and $1.58 \mathrm{~cm}$, respectively, which were not significantly different.

the present study is the first to assess the differences in the CT measurement of cancer thickness with or without gastric filling.

The stomach is a hollow organ, and anatomical studies $(20,21)$ undertaken long before CT scanning was used to examine the stomach revealed that the thickness of the normal gastric wall is associated with the degree of filling of the abdominal cavity, which is concordant with the findings of the present study. The thickness of the normal gastric wall was $>5 \mathrm{~mm}$ when the stomach was empty, among which the thickness at the greater curvature was $>10 \mathrm{~mm}$, as the duplicatures at this area are thick and shrinking. However, once the abdominal cavity was full, the thickness of the gastric wall at each area was $<5 \mathrm{~mm}$. In addition, the effects of gastric filling degree on the thickness of the gastric wall at the greater curvature were the highest. However, the effects of filling on the thickness of gastric wall at cardia and antrum were relatively low, which could be associated with the thicker duplicatures at the greater curvature, whereas the muscular layer is thinner. Therefore, the duplicatures gather together when the stomach is empty, and are stretched following filling. By contrast, the muscular layers at the cardia and antrum are relatively thick, and thus their extensibility is limited. Therefore, a criterium stating that 'the thickness of gastric wall $>5 \mathrm{~mm}$ is considered as gastric wall thickening' is not necessarily applicable for all the gastric areas in clinical imaging evaluation $(22,23)$. The filling degree of the stomach and the gastric area to be evaluated should therefore be considered when using the criteria.

In the present study, to reduce the effects of changes to the tumors on the measurements, and to avoid increasing 
Table III. Comparison of the measured thickness of the gastric carcinomas prior to and post-filling.

\begin{tabular}{lccccc}
\hline Area & Patients, $\mathrm{n}$ & Thickness prior to filling, $\mathrm{cm}^{\mathrm{a}}$ & Thickness post-filling, $\mathrm{cm}^{\mathrm{a}}$ & Z-value & P-value \\
\hline Newly diagnosed & & & & & \\
$\quad$ Total & 32 & $1.47(1.20,1.76)$ & $1.50(1.18,1.75)$ & -1.660 & 0.097 \\
Cardia & 11 & $1.25(1.06,1.73)$ & $1.26(1.01,1.58)$ & -0.868 & 0.386 \\
Lesser curvature & 13 & $1.47(0.94,1.74)$ & $1.45(0.98,1.72)$ & -0.654 & 0.513 \\
Greater curvature & 2 & $1.42(1.24,1.59)$ & $1.42(1.28,1.55)$ & - & $-{ }^{\mathrm{b}}$ \\
Antrum & 6 & $2.17(1.47,4.36)$ & $2.15(1.48,4.11)$ & -1.577 & 0.115 \\
Re-examination & & & & & \\
Total & 31 & $1.76(1.20,2.31)$ & $1.23(0.87,1.87)$ & -4.861 & $<0.001$ \\
Cardia & 13 & $1.86 \pm 0.87$ & $1.58 \pm 0.96$ & 2.591 & 0.024 \\
Lesser curvature & 8 & $1.81 \pm 0.35$ & $1.41 \pm 0.70$ & 2.999 & 0.020 \\
Greater curvature & 3 & $2.57(1.10,2.58)$ & $1.05(0.90,1.68)$ & - & $-\mathrm{b}$ \\
Antrum & 7 & $1.73 \pm 0.51$ & $1.34 \pm 0.57$ & 3.224 & 0.018
\end{tabular}

${ }^{\mathrm{a}}$ Data are presented as the mean \pm standard deviation or median ( $95 \%$ confidence interval). ${ }^{\mathrm{b}}$ As the number of gastric carcinoma at the greater curvature was low ( 2 carcinomas in the newly diagnosed patients and 3 in the patients undergoing re-examination), the thickness of the gastric carcinoma at the greater curvature prior to and following gastric filling was not compared.

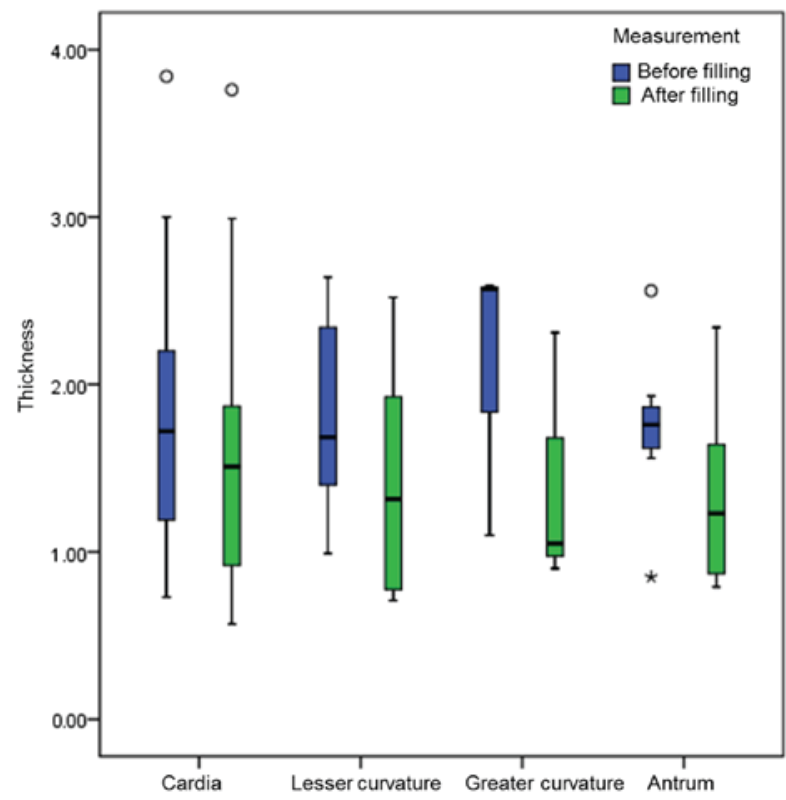

Figure 4. Thickness of the gastric cancer at each area prior to and following gastric filling in the re-examination group of patients.

irradiation doses for patients, plain CT scanning was adopted when the stomach was empty, and enhanced scanning was adopted subsequent to filling. Therefore, the measurements prior to and following filling could be obtained in one examination. Although the scanning parameters were different in the two measurements, the density of the soft tissues of gastric cancer was evidently different from that of the intra-gastric gas and extra-gastric adipose tissue, thus plain CT scanning could clearly measure the boundaries. In addition, the methods of gastric cancer thickness measurement were determined: For instance, the layer with the highest gastric cancer thickness was selected, the regions adhered to perigastric lymph nodes were avoided, and perigastric adipose tissues with evidence of

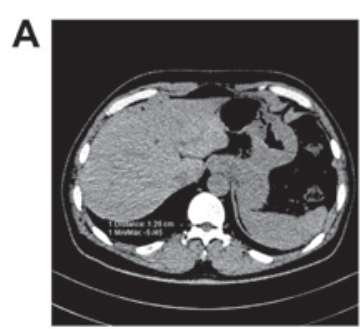

B
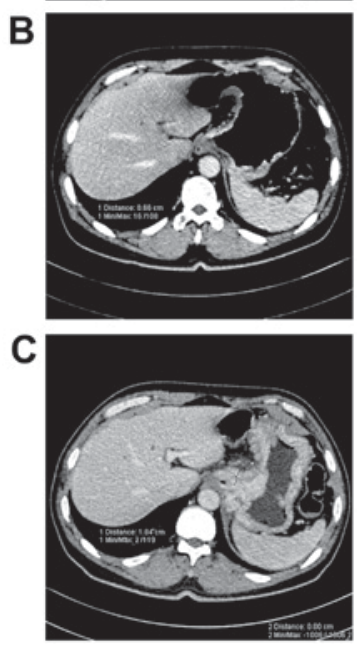

Figure 5. A 61-year-old male patient with cardiac cancer who underwent re-examination at 3 months following neoadjuvant chemotherapy. The thickness of the cardiac cancer (A) prior to and (B) following filling was 1.29 and $0.66 \mathrm{~cm}$, respectively, which were significantly different. Compared with the cancer thickness (C) prior to treatment in the CT image (thickness, $1.84 \mathrm{~cm}$ ), the thickness following treatment reduced significantly. The postoperative pathological results showed tumor regression following treatment was grade I.

invasion were not included in the measurements. According to the locations of the measurements in the venous phase, the same locations were selected in the plain scanning images using anatomical landmarks of the gastric wall, including the 


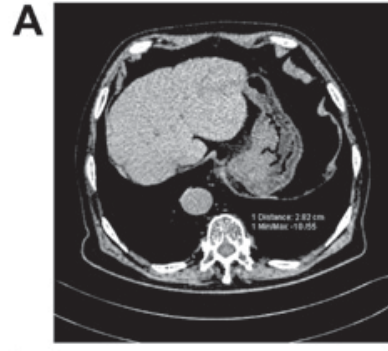

B
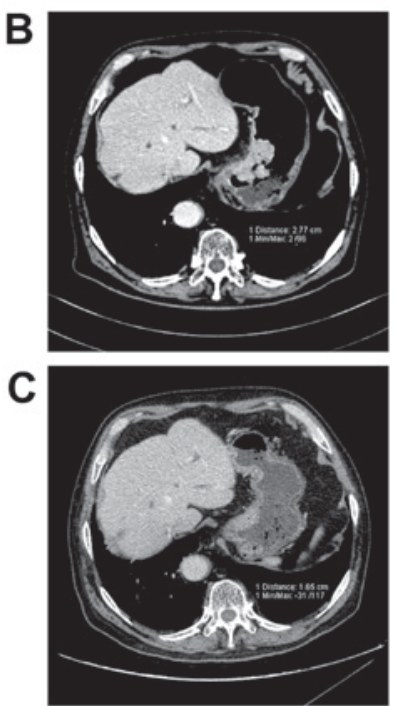

Figure 6. A 69-year-old male patient with cardiac cancer for re-examination at 8 months following non-surgical treatment. The thickness of the cardiac cancer (A) prior to and (B) following filling was 2.82 and $2.77 \mathrm{~cm}$, respectively, which were not significantly different. Comparing with the cancer thickness prior to treatment in the CT image $(C$, thickness $=1.65 \mathrm{~cm})$, the thickness once the treatment increased.

cardia, pylorus and ulcer floor, rather than adjacent organs or selected layers. Therefore, the thicknesses measured by these two different scanning procedures were comparable. To avoid measurement errors in CT assessment of the depth of gastric cancer invasion, as it is widely accepted that CT is unable to measure either the depth of early-stage gastric cancer or the effects of the rest of normal tissue situated in deep layer on the measurement of the gastric cancer wall depth, all patients enrolled in the present study had advanced gastric cancer with tumor invasion through the whole gastric wall under CT. The thickness of gastric cancer was 50\% higher than the thickness of the adjacent normal gastric wall following filling. Therefore, the technique used in the present study could not be applied to all patients with gastric cancer. However, the technique was suitable for gastric cancer in any region of the stomach, which met the inclusion criteria. The present study included cases of cardia cancer.

The findings of the present study revealed that the thickness of a gastric cancer is not affected by the degree of gastric filling in newly diagnosed patients. However, for the patients who were undergoing re-examination following non-surgical treatment, the gastric cancer thickness could change with the degree of filling. These changes could be associated with the more evident muscular layer involvement, deeper gastric wall invasion and denser cancerous tissues in newly diagnosed advanced gastric cancer patients. However, necrosis, fibrosis, and shallower gastric wall invasion were observed

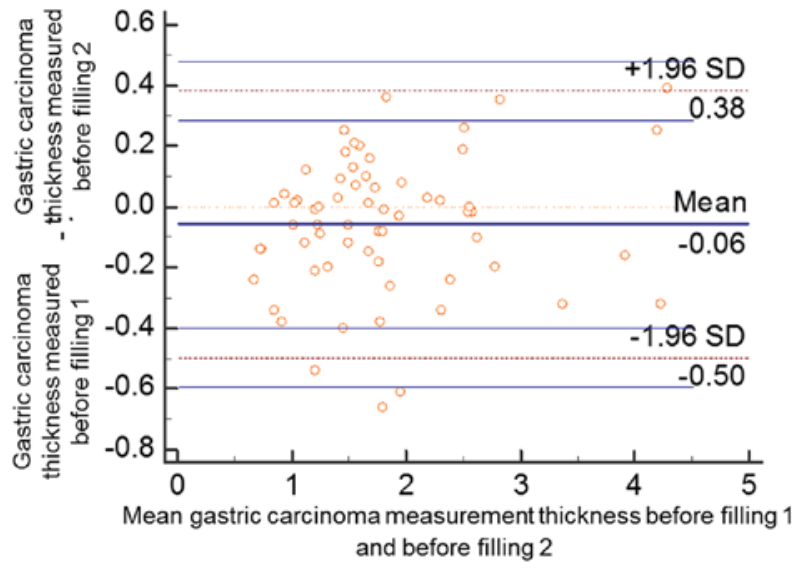

Figure 7. The Bland and Altman plot of the two measurements of gastric cancer thickness prior to filling.

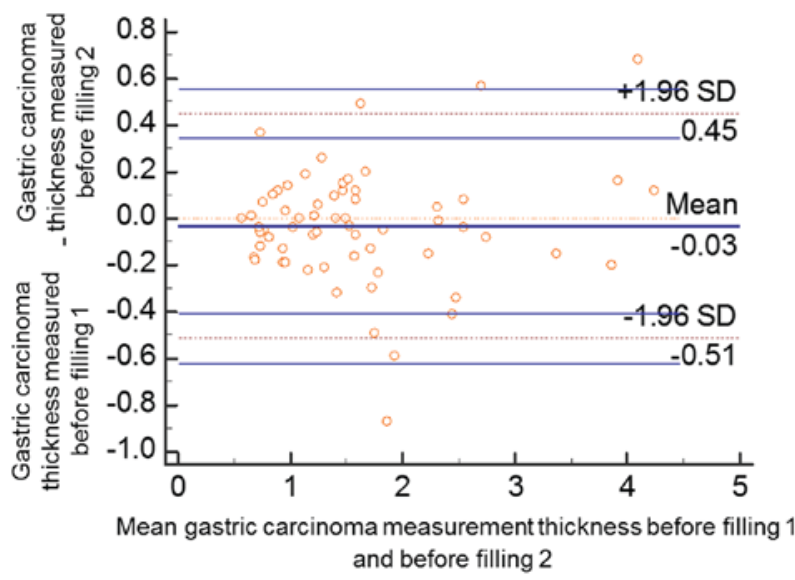

Figure 8. The Bland and Altman plot of the two measurements of gastric cancer thickness post-filling.

in the patients who had received non-surgical treatments. Therefore, we hypothesized that the thickness of advanced gastric cancer remains relatively unchanged upon gastric filling prior to treatment, and could therefore be used as the baseline measurement. However, the degree of filling of the stomach should be consistent between the initial examination and re-examination, which could aid the accurate assessment of the treatment efficacy. The compliance of patients with gastric cancer with the oral intake of a large volume of liquid filling agent is lower than that of the general population. Thus, the dose of oral liquid filling agent used in the two examinations among different patients, and even in the same patient, could differ, as it is difficult to maintain an identical degree of gastric filling between the two measurements. As with liquid, gas has also been widely used in clinical practices as a negative filling agent, and its use has been reported in several previous studies $(1,24-29)$. When mixed with liquid, aerogenic powder generates carbon dioxide, which is non-toxic and non-hazardous. In the present study, two packs of aerogenic powder $(6 \mathrm{~g})$ were used for each patient, which released $800-1,000 \mathrm{ml}$ gas, which was similar to the dose of oral liquid filling agent. Compared with the liquid filling agent, a gas filling agent has several advantages. i) Prior to CT scanning, 
the degree of gastric filling could be determined according to the positioning image. For patients with a sub-optimal filling state, the filling agent could be added. ii) The compliance and tolerability of patients was superior when using a gas filling agent. Abdominal fullness occurred in certain patients, which could be alleviated by belching or exhausting. Compared with a liquid filling agent, a gas filling agent is more easily excreted, and no complications, including perforation, have been reported. iii) gas can be more homogeneously distributed than liquid, and the dilation of gastric wall is more even, thus the position of patients did not require changing during the examination. iv) The volume of gas generated by single unit of aerogenic powder is relatively stable, which aided control of the degree of filling of the abdominal cavity. Therefore, it is easier for clinicians to maintain the filling degree of the abdominal cavity at the same level, and thus aid evaluation of treatment efficacy. v) It could aid the performance of CT virtual endoscopy and thus facilitate the early detection and display of a gastric cancer. Therefore, when performing gastric CT scanning, particularly for patients with gastric cancer who have undergone non-surgical treatments, fixed-dose gas filling should be applied to aid the consequent evaluation of treatment efficacy.

While performing evaluations of treatment efficacy in clinical practices, the same investigator is generally asked to conduct the measurements prior to and following treatments. Therefore, in the present study the same investigator repeated the measurements 1 month later to assess the reproducibility of measuring gastric cancer thickness, following which Bland and Altman plots were produced to assess consistency. The findings of this study showed that $>95 \%$ of all data points prior to and following filling were in the $95 \%$ CI of LoA, indicating that the two measurements were highly consistent. Therefore, measuring the thickness of gastric lesions has relatively high reproducibility and could be used as one of the parameters for the imaging measurement.

The CT images of the patients were also retrospectively reviewed prior to treatment, and it was found that for the patients that the gastric cancer thickness was greatly affected by the filling degree, the thickness generally decreased following the treatment (Fig. 5). For the patients for whom the gastric cancer thickness was slightly affected by the filling degree, the thickness generally remained unchanged or even increased following the treatment (Fig. 6). As certain patients underwent a liquid filling method at their initial examination, the filling degree of the abdominal cavity could differ, thus the second measurement was not obtained for these patients and the consequent statistical analyses were not performed. These findings indicated that the differences in gastric cancer thickness prior to and following treatment could be associated with the treatment efficacy.

There are several limitations to the present study. First, because the method requires the tumors to be recognized in plain CT images prior to gastric filling, the patients included in the present study generally exhibited high gastric cancer thickness and advanced disease stage, and there was a lack of T2-stage patients. Second, the present study only assessed the effects of gastric filling degree on the measurement of the thickness of gastric cancer; the effects on the area or the longest diameter of the cancer were not investigated. Third, the effects of gastric filling degree on different pathological types of gastric cancer were not compared. Finally, certain patients undergoing re-examination used liquid filling prior to treatment, and the treatment efficacies were not confirmed by pathological results. Therefore, more studies are required to investigate the clinical significance of measuring gastric cancer thickness on treatment efficacy evaluation.

In conclusion, the measured thickness of gastric cancer in newly diagnosed patients was relatively stable and may be used as an indicator in baseline CT examination. Maintaining a similar degree of gastric filling during re-examination may help to accurately evaluate treatment efficacy.

\section{Acknowledgements}

Not applicable.

\section{Funding}

No funding was received.

\section{Availability of data and materials}

The datasets used and/or analyzed during the current study are available from the corresponding author on reasonable request.

\section{Authors' contributions}

YL designed the research. JL and GW collected clinical data and performed computed tomography scans. LY and GS performed the experiments. TZ and JP analyzed the data. LY, YL and GS wrote the manuscript.

\section{Ethics approval and consent to participate}

Application Form of Ethical Review from The Fourth Hospital of Hebei Medical University (approval no. 2016 MEC111).

\section{Consent for publication}

Patients, parents or guardians provided written informed consent for the publication of the present study.

\section{Competing interest}

The authors declare that they have no competing interests.

\section{References}

1. Hallinan JT and Venkatesh SK: Gastric carcinoma: Imaging diagnosis, staging and assessment of treatment response. Cancer Imaging 13: 212-227, 2013.

2. Carl-Mcgrath S, Ebert M and Röcken C: Gastric adenocarcinoma: Epidemiology, pathology and pathogenesis. Cancer Therapy 6: 877-893, 2008.

3. Zuo T, Zheng R, Zeng H, Zhang S and Chen W: Epidemiology of stomach cancer in China. Chin J Clin Oncol 44: 52-58, 2017.

4. Ghiţă D, Glavici A, Pleşea IE, Săftoiu A, Dumitrescu D and Ciurea T: Invasion assessment in gastric carcinoma-imagistic and histopathologic combined study. Rom J Morphol Embryol 52 (Suppl 1): S349-S361, 2011. 
5. Moschetta M, Stabile Ianora AA, Anglani A, Marzullo A, Scardapane A and Angelelli G: Preoperative T staging of gastric carcinoma obtained by MDCT vessel probe reconstructions and correlations with histological findings. Eur Radiol 20: 138-145, 2010.

6. Kim JH, Eun HW, Hong SS, Kim YJ, Han JK and Choi BI: Gastric cancer detection using MDCT compared with 2D axial CT: Diagnostic accuracy of three different reconstruction techniques. Abdom Imaging 37: 541-548, 2012.

7. Bhandari S, Shim CS, Kim JH, Jung IS, Cho JY, Lee JS, Lee MS and Kim BS: Usefulness of three-dimensional, multidetector row CT (virtual gastroscopy and multiplanar reconstruction) in the evaluation of gastric cancer: A comparison with conventional endoscopy, EUS, and histopathology. Gastrointest Endosc 59: 619-626, 2004

8. Furukawa K, Miyahara R, Itoh A, Ohmiya N, Hirooka Y, Mori K and Goto H: Diagnosis of the invasion depth of gastric cancer using MDCT with virtual gastroscopy: Comparison with staging with endoscopic ultrasound. AJR Am J Roentgenol 197: 867-875, 2011.

9. Washington K: 7th Edition of the AJCC Cancer Staging Manual: Stomach. Ann Surg Oncol 17: 3077-3079, 2010.

10. DI Cicilia R, Mordenti P, Anselmi E, Paties C, Carella E and Cavanna L: HER2-positive gastric cancer showing marked thickening of the gastric wall on ultrasonographic and computed tomographic scans. A chance phenomenon or a specific behaviour of this cancer type? Report of three cases. Anticancer Res 34: 5083-5086, 2014.

11. Lee SM, Kim SH, Lee JM, Im SA, Bang YJ, Kim WH, Kim MA, Yang HK, Lee HJ, Kang WJ, et al: Usefulness of CT volumetry for primary gastric lesions in predicting pathologic response to neoadjuvant chemotherapy in advanced gastric cancer. Abdom Imaging 34: 430-440, 2009.

12. Beer AJ, Wieder HA, Lordick F, Ott K, Fischer M, Becker K, Stollfuss J and Rummeny EJ: Adenocarcinomas of esophagogastric junction: Multi-detector row CT to evaluate early response to neoadjuvant chemotherapy. Radiology 239: 472-480, 2006.

13. Wieder HA, Beer AJ, Lordick F, Ott K, Fischer M, Rummeny EJ, Ziegler S, Siewer JR, Schwaiger M and Weber WA: Comparison of changes in tumor metabolic activity and tumor size during chemotherapy of adenocarcinomas of the esophagogastric junction. J Nucl Med 46: 2029-2034, 2005.

14. Horton KM and Fishman EK: Current role of $\mathrm{CT}$ in imaging of the stomach. Radiographics 23: 75-87, 2003.

15. Gligorievski A: CT evaluation of gastric lymphoma. Prilozi 30: 125-138, 2009

16. Giuliani A, Caporale A, Di Bari M, Demoro M, Gozzo P, Corona M, Miccini M, Ricciardulli T and Tocchi A: Maximum gastric cancer diameter as a prognostic indicator: Univariate and multivariate analysis. J Exp Clin Cancer Res 22: 531-538, 2003.

17. Ahn HS, Kim SH, Kodera Y and Yang HK: Gastric cancer staging with radiologic imaging modalities and UICC staging system. Dig Surg 30: 142-149, 2013.
18. Kim JW, Shin SS, Heo SH, Choi YD, Lim HS, Park YK, Park CH, Jeong YY and Kang HK: Diagnostic performance of 64-section $\mathrm{CT}$ using $\mathrm{CT}$ gastrography in preoperative $\mathrm{T}$ staging of gastric cancer according to 7th edition of AJCC cancer staging manual. Eur Radiol 22: 654-662, 2012.

19. Kajitani T: The general rules for gastric cancer study in surgery and pathology. Part I. Clinical classification. Jpn J Surg 11: $127-139,1981$.

20. Miederer SE and Schepp W: The mucosal barrier of the stomach. Anatomic structure and function. Dtsch Med Wochenschr 110: 852-856, 1985 (In German)

21. Lim JH and Jeong YM: Sonography of the stomach: An in vitro study to determine the anatomic cause of inner hyperechoic and hypoechoic layers of the gastric wall. AJR Am J Roentgenol 162: 335-338, 1994.

22. Kim HJ, Kim AY, Oh ST, Kim JS, Kim KW, Kim PN, Lee MG and Ha HK: Gastric cancer staging at multi-detector row CT gastrography: Comparison of transverse and volumetric CT scanning. Radiology 236: 879-885, 2005.

23. Fukuya T, Honda H, Kaneko K, Kuroiwa T, Yoshimitsu K, Irie H, Maehara Y and Masuda K: Efficacy of helical CT in T-staging of gastric cancer. J Comput Assist Tomogr 21: 73-81, 1997.

24. Tang L, Li ZY, Li ZW, Zhang XP, Li YL, Li XT, Wang ZL, Ji JF and Sun YS: Evaluating the response of gastric carcinomas to neoadjuvant chemotherapy using iodine concentration on spectral CT: A comparison with pathological regression. Clin Radiol 70: 1198-1204, 2015.

25. Kumano S, Okada M, Shimono T, Kuwabara M, Yagyu Y, Imaoka I, Ashikaga R, Ishii K and Murakami T: T-staging of gastric cancer of air-filling multidetector-row CT: Comparison with hydro-multidetector-row CT. Eur J Radiol 81: 2953-2960, 2012.

26. Moschetta M, Scardapane A, Telegrafo M,Lorusso V, Angelelli G and Stabile Ianora AA: Differential diagnosis between benign and malignant ulcers: 320 -row CT virtual gastroscopy. Abdom Imaging 37: 1066-1073, 2012.

27. Yen PP and Stevenson G: Two- and three-dimensional examination of the stomach (virtual gastroscopy): Technical note. Can Assoc Radiol J 61: 41-43, 2010

28. Okten RS, Kacar S, Kucukay F, Sasmaz N and Cumhur T: Gastric subepithelial masses: Evaluation of multidetector CT (multiplanar reconstruction and virtual gastroscopy) versus endoscopic ultrasonography. Abdom Imaging 37: 519-530, 2012.

29. Takahashi S, Hirayama M, Kuroiwa G, Kawano Y, Takada K, Sato T, Miyanishi K, Sato Y, Takimoto R, Kobune M and Kato J: Diagnostic validity of CT gastrography versus gastroscopy for primary lesions in gastric cancer: Evaluating the response to chemotherapy, a retrospective analysis. Gastric Cancer 16: 543-548, 2013.

This work is licensed under a Creative Commons Attribution-NonCommercial-NoDerivatives 4.0 International (CC BY-NC-ND 4.0) License. 